\title{
Guizhi Fuling Wan as a Novel Agent for Intravesical Treatment for Bladder Cancer in a Mouse Model
}

\author{
Chi-Chen Lu, ${ }^{1,2,3^{*}}$ Cheng-Huang Shen, ${ }^{4,5^{*}}$ Chia-Bin Chang, ${ }^{2,3}$ Hsiao-Yen Hsieh, ${ }^{2,3,4}$ Jiann-Der Wu, ${ }^{6}$ Ling-Huei Tseng, ${ }^{2}$ \\ Dennis W Hwang, ${ }^{7}$ Syue-Yi Chen, ${ }^{4}$ Shu-Fen Wu, ${ }^{2,3}$ Michael W Y Chan, ${ }^{2,3}$ and Cheng-Da Hsu ${ }^{2,4,5}$
}

${ }^{1}$ Department of Chinese Medicine, Ditmanson Medical Foundation Chia-Yi Christian Hospital, Chia-Yi, Taiwan; ${ }^{2}$ Graduate Institute of Molecular Biology, National Chung Cheng University, Chia-Yi, Taiwan; ${ }^{3}$ Department of Life Science, National Chung Cheng University, Chia-Yi, Taiwan; Departments of ${ }^{4}$ Medical Research, ${ }^{5}$ Urology, and ${ }^{6}$ Pathology, Ditmanson Medical Foundation Chia-Yi Christian Hospital, Chia-Yi, Taiwan; and ${ }^{7}$ Department of Chemistry and Biochemistry, National Chung Cheng University, Chia-Yi, Taiwan

\begin{abstract}
Alternative intravesical agents are required to overcome the side effects currently associated with the treatment of bladder cancer. This study used an orthotopic bladder cancer mouse model to evaluate Guizhi Fuling Wan (GFW) as an intravesical agent. The effects of GFW were compared with those of mitomycin-C (Mito-C) and bacille Calmette-Guérin (BCG). We began by evaluating the response of the mouse bladder cancer cell line MB49 to GFW treatment, with regard to cell viability, cell cycle progression and apoptosis. MB49 cells were subsequently implanted into the urothelial walls of the bladder in female C57BL/6 mice. The success of the model was confirmed by the appearance of hematuria and tumor growth in the bladder. Intravesical chemotherapy was administered in accordance with a published protocol. In vitro data revealed that GFW arrested MB49 cell cycle in the G0/G1 phase, resulting in the suppression of cell proliferation and induced apoptosis. One possible mechanism underlying these effects is an increase in intracellular reactive oxygen species (ROS) levels leading to the activation of ataxia telangiectasia-mutated (ATM)/checkpoint kinase 2 (CHK2) and ATM/P53 pathways, thereby mediating cell cycle progression and apoptosis, respectively. This mouse model demonstrates the effectiveness of GFW in the tumor growth, with results comparable to those achieved by using BCG and Mito-C. Furthermore, GFW was shown to cause only mild hematuria. The low toxicity of the compound was confirmed by a complete lack of lesions on bladder tissue, even after 10 consecutive treatments using high concentrations of GFW. These results demonstrate the potential of GFW for the intravesical therapy of bladder cancer.
\end{abstract}

Online address: http://www.molmed.org

doi: $10.2119 / \mathrm{molmed} .2015 .00085$

\section{INTRODUCTION}

Urothelial carcinoma is the second most common cause of death among genitourinary tumors (1). More than $90 \%$ of urothelial carcinoma is transitional cell carcinoma, $20 \%$ of which develops into muscle invasive disease with a substantial risk of distant metastasis (2). Despite the fact

${ }^{*} \mathrm{C}-\mathrm{CL}$ and $\mathrm{C}-\mathrm{HS}$ contributed equally to this work.

Address correspondence to Cheng-Da Hsu, Department of Medical Research, Ditmanson Medical Foundation, Chia-Yi Christian Hospital, 539 Zhongxiao Road, East District, Chiayi City 600, Taiwan. Phone: +886-5-2765041, ext. 5558; Fax: +886-5-2765041, ext. 5555; E-mail: cych06390@gmail.com; or Michael WY Chan, Room 452, Department of Life Science, National Chung Cheng University, 168 University Road, Min-Hsiung, Chia-Yi, Taiwan. Phone: +886-5-2720411, ext. 66510; Fax: +886-5-2722871; E-mail: biowyc@ccu.edu.tw; or Shu-Fen Wu, Room 452, Department of Life Science, National Chung Cheng University, 168 University Road, Min-Hsiung, Chia-Yi, Taiwan. Phone: +886-5-2720411, ext. 66509; Fax: +886-5-2722871; E-mail: biosfw@ccu.edu.tw. Submitted April 12, 2015; Accepted for publication January 13, 2016; Published Online (www.molmed.org) January 13, 2016.

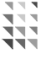

Feinstein Institute

for Medical Research Northwell Health

that urothelial carcinoma can originate in any part of the urinary tract, most cases begin in the urinary bladder (3). Bladder urothelial carcinoma is heterogeneous and can be histologically subdivided into low-grade and high-grade diseases. Low-grade urothelial carcinoma is uniformly noninvasive; however, high-grade urothelial carcinoma can be classified according to the depth of invasion into the muscle layer, as follows: nonmuscle invasive bladder cancer (Tis, Ta, T1) and muscle-invasive bladder cancer $(\geq \mathrm{T} 2)(4)$. Patients diagnosed with nonmuscle invasive bladder cancer face a high risk of recurrence (5), which necessitates the additional use of intravesical chemotherapy or bacille Calmette-Guérin (BCG) as a supplement to transurethral resection $(6,7)$. However, mitomycin-C (Mito-C) has been shown to induce 
cystitis and hematuria $(8,9)$, and BCG is associated with cystitis and granulomatous inflammation $(10,11)$. Thus, novel intravesical agents with enhanced efficacy and minimal toxicity are required for the treatment of patients with bladder cancer.

The traditional Chinese herbal formula, Guizhi Fuling Wan (GFW), has recently been shown to have anticancer effects on hepatocellular carcinoma $(12,13)$ and cervical cancer (14). We have previously shown that GFW has a potent inhibitory effect on cell proliferation by inducing cell cycle arrest and apoptosis in human bladder cancer cell lines, BFTC 905 and TSGH 8301, with only weak cytotoxicity effects on normal human urothelial cells (15).

Recent studies have identified several traditional Chinese herbal remedies capable of inducing apoptosis in cancer cells by increasing the level of reactive oxygen species (ROS) (16-18). ROS are oxygen-containing chemically reactive molecules that can cause considerable damage to key cellular molecules, such as lipids, proteins and DNA $(19,20)$. ROS-induced DNA damage exceeding cellular repair capacity can lead to apoptosis. Furthermore, the activation of ataxia telangiectasia-mutated (ATM) kinase signaling and tumor suppressor p53 was identified as an important cellular response to ROS-induced DNA damage $(21,22)$. The generation of excess ROS has also been shown to initiate cell cycle arrest, which can lead to the induction of p53-dependent apoptosis (23). This study sought to identify the mechanism underlying ROS involvement in the GFW-related suppression of cell growth and induced cell death in bladder cancer. We also applied a murine orthotopic bladder tumor model to evaluate the in vivo antitumor effects of intravesical GFW and compare the treatment outcomes with those obtained using Mito-C or BCG.

\section{MATERIALS AND METHODS}

\section{Preparation of Agents and Cell Culture}

GFW herbal extract was purchased, validated and prepared as previously described (15). Mito-C (Kyowa Hakko Kirin) and BCG (Sanofi Pasteur Limited) were dissolved in normal saline buffer at $1 \mathrm{mg} / \mathrm{mL}$ to provide stock solutions, which were then diluted to $0.4 \mathrm{mg} / \mathrm{mL}$ and $1 \times 10^{8} \mathrm{CFU} / 3 \mathrm{~mL}$, respectively. Murine urothelial carcinoma MB49 cells (gift from Dr. Timothy L. Raliff, Purdue Cancer Center) were cultured in RPMI 1640 supplemented with $10 \%$ fetal bovine serum (FBS), 100 units/mL penicillin and $100 \mu \mathrm{g} / \mathrm{mL}$ streptomycin in an incubator in a humidified atmosphere containing $5 \% \mathrm{CO}_{2}$ at $37^{\circ} \mathrm{C}$.

\section{Cell Cycle Analysis}

MB49 cells were seeded in six-well plates at $2 \times 10^{5}$ cells per well and cultured for $24 \mathrm{~h}$, before being starved in medium without supplemental FBS for $24 \mathrm{~h}$, followed by treatment with 0.5, 1 and $2 \mathrm{mg} / \mathrm{mL}$ GFW for $24 \mathrm{~h}$. After treatment, the cells were trypsinized, washed with phosphate-buffered saline (PBS) and fixed by using methanol at $4^{\circ} \mathrm{C}$ overnight, followed by incubation with RNase A and propidium iodide (Sigma) for $30 \mathrm{~min}$ at room temperature. The cells were then analyzed by using a BD FACSCanto II flow cytometer (BD Biosciences) in conjunction with Mod Fit LT' 3.3 software.

\section{Apoptosis Analysis}

MB49 cells were seeded in six-well plates at $2 \times 10^{5}$ cells per well and cultured for $24 \mathrm{~h}$ before being treated with $0.5,1$ or $2 \mathrm{mg} / \mathrm{mL}$ of GFW for $24 \mathrm{~h}$. Cell apoptosis was detected using the Annexin V-FITC Apoptosis Detection Kit (BioVision) in accordance with the manufacturer's protocol and analyzed by BD FACSCanto II flow cytometer (BD Biosciences) in conjunction with Mod Fit LT ${ }^{\mathrm{TM}} 3.3$ software.

\section{Reactive Oxygen Species Detection}

This assay used cell-permeable fluorogenic probe 2', 7'-dichlorodihydrofluorescein diacetate (DCFH-DA). DCFH-DA can be deacetylated by using cellular esterases to nonfluorescent DCFH, which can be rapidly oxidized to highly fluorescent
DCF by intracellular ROS. The intensity of fluorescence is proportional to the intracellular ROS levels.

Briefly, MB49 cells were seeded in 10 -cm plates at $2 \times 10^{6}$ cells per plate and cultured for $24 \mathrm{~h}$. The cells were starved in medium without supplemental FBS for $24 \mathrm{~h}$ before being pre-incubated with $20 \mu \mathrm{mol} / \mathrm{L}$ DCFH-DA for $1 \mathrm{~h}$. This step was then followed by treatment with $2 \mathrm{mg} / \mathrm{mL}$ GFW (experimental group) or $50 \mu \mathrm{mol} / \mathrm{L} \mathrm{H}_{2} \mathrm{O}_{2}$ (positive control group) for $3 \mathrm{~h}$. After collecting and washing the cells with PBS, they were immediately analyzed by using BD FACSCanto II flow cytometer (BD Biosciences) in conjunction with Mod Fit LT $^{\text {TM }} 3.3$ software.

\section{Western Blot Analysis}

MB49 cells were seeded in six-well plates at $2 \times 10^{5}$ cells per well and cultured for $24 \mathrm{~h}$. The cells were starved in medium without supplemental FBS for $24 \mathrm{~h}$ and then treated with $2 \mathrm{mg} / \mathrm{mL}$ GFW for various durations. Total cellular proteins were extracted, and the protein concentrations in the extracts were determined by using the Bio-Rad Protein Assay reagent (Bio-Rad) with bovine serum albumin as a standard. Each cell lysate (30 $\mu \mathrm{g})$ was separated on SDS-PAGE, transferred electrophoretically to polyvinylidene fluoride transfer membranes and probed by using antibodies - 1:1,000 diluted antibody against caspase-3, ATM, CHK2, phospho-CHK2, P53, phospho-P53 and P21 (Cell Signaling). The protein band that was specifically bound to the primary antibody was detected by using the BioSpectrum 800 system (UVP) after incubating with horseradish peroxidase (HRP)-conjugated secondary antibodies for $1.5 \mathrm{~h}$ at room temperature.

\section{Murine Orthotopic Bladder Tumor Model and Intravesical Therapy}

Female C57BL/ 6 mice aged 6 wks were provided by the National Laboratory Animal Center and maintained at 
our animal care facility for $2 \mathrm{wks}$ before use. All experiments were approved by the Institutional Animal Care and Use Committee of National Chung Cheng University (IACUC no. 1020103).

The implantation of MB49 murine bladder cancer cells into C57BL/6 mice was performed as previously described (2) with only slight modifications. Mice were anaesthetized through the administration of $1.5 \%$ Isoflurane gas, whereupon a 24-gauge Teflon intravenous catheter (BD Biosciences) was inserted through the urethra into the bladder. In preparation for tumor implantation,

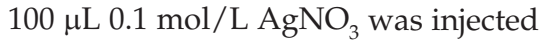
into the bladder to induce a chemical lesion on the bladder wall. After $10 \mathrm{~s}$, the content was washed out through the transurethral infusion of $1 \mathrm{~mL}$ PBS. A suspension of $5 \times 10^{5} / 100 \mu \mathrm{L}$ MB49 cells was then instilled into the bladder. The mice were randomly assigned to five groups (seven mice per group). Twenty-four hours after tumor implantation, the experimental group underwent intravesical therapy with $100 \mu \mathrm{L}$ GFW (180 mg/mL), $100 \mu \mathrm{L}$ Mito-C (0.4 mg/mL) or PBS every other day, or $100 \mu \mathrm{L} \mathrm{BCG}\left(1 \times 10^{8} \mathrm{CFU} / 3 \mathrm{~mL}\right)$ therapy every week (Figure 1A). At the same time, the control group received no treatment (Figure 1B). After injection of GFW, Mito-C, BCG or PBS into the mouse bladders, the external urethral orifice of each mouse was blocked for $1 \mathrm{~h}$ by using a small piece of Micropore surgical tape. The tape was subsequently removed, and the mice were returned to their original cages. Healthy mice that did not undergo tumor implantation were used as a control to evaluate the cytotoxic effects of GFW on the epithelium of a normal bladder. GFW treatment was administered 10 times every other day to healthy mice with or without $\mathrm{AgNO}_{3}$-induced lesion.

\section{In Vivo Magnetic Resonance Detection of Bladder Tumors}

Murine bladder tumor cells implanted into mouse bladders (as described above) were scanned by using magnetic resonance imaging (MRI) on d 0, 4, 7 and 11 after GFW treatment. MRI data were acquired by using a 300-MHz (7 T) Apollo NMR spectrometer (Tecmag) capable of microimaging. Phantom images were obtained by using microimaging probe heads and a 32-mm I.D. imaging RF coil (Doty Scientific) with a self-shielded gradient system and maximum strength of $1,000 \mathrm{mT} / \mathrm{m}$ in the $\mathrm{x}, \mathrm{y}$ and $\mathrm{z}$ directions (Resonance Research). All MRI data were generated by using Spin-Echo pulse sequences. The echo time (TE) and repetition time (TR) were $15 \mathrm{~ms}$ and $7 \mathrm{~s}$, respectively.

\section{Statistical Analysis}

Numerical data are expressed as either mean \pm standard error of the mean (SEM) or as a percentage relative to the untreated control. Differences between the experiment and control groups were determined by using one-way analysis of variance (ANOVA) followed by Dunnett test. Differences were considered significant when the $p$ value was $<0.05$ or within the $95 \%$ confidence interval.
All supplemental materials are available online at www.molmed.org.

\section{RESULTS}

\section{GFW Inhibits MB49 Cell Proliferation through Cell Cycle Arrest and Induced Apoptosis}

In a previous study, we demonstrated the inhibitory effects of GFW on the proliferation of human bladder cancer cells with minimal toxicity to normal urothelial cells. In this study, we sought to determine the effectiveness of GFW in the treatment of mouse bladder cancer cell line MB49. The initial results of in vitro cell viability assays revealed that GFW suppresses the proliferation of MB49 cells in a dosage-dependent manner, presenting a half-maximal inhibitory concentration $\left(\mathrm{IC}_{50}\right.$ ) of approximately $0.95 \mathrm{mg} / \mathrm{mL}$ (Supplementary Figure S1), in accordance with its effect on human bladder cancer cell line TSGH 8301 (15).
A

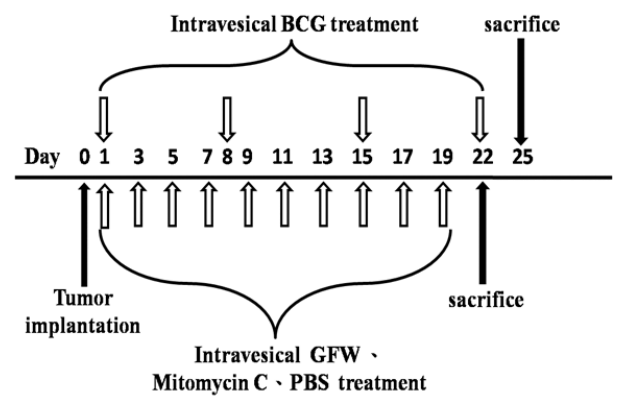

B

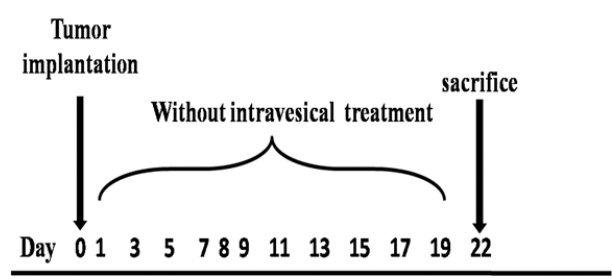

Figure 1. Strategy used in intravesical treatment in a murine orthotopic bladder tumor model. Time schedules of treatment groups (A) and nontreatment control groups (B) are shown. Twenty-four hours after tumor implantation, animals in the treatment group underwent intravesical therapy with $100 \mu \mathrm{L}$ GFW (180 mg/mL), $100 \mu \mathrm{L}$ Mito-C $(0.4 \mathrm{mg} / \mathrm{mL})$ or PBS every other day (empty arrows pointing up), or $100 \mu \mathrm{L} \mathrm{BCG}\left(1 \times 10^{8} \mathrm{CFU} / 3 \mathrm{~mL}\right)$ therapy every week (empty arrows pointing down), while the control group received no treatment. 
To identify the mechanism by which GFW inhibits the growth of MB49 cells, we sought to determine whether GFW treatment hinders the cell cycle progression. Flow cytometry results demonstrated that exposure to GFW at 1 and $2 \mathrm{mg} / \mathrm{mL}$ for $24 \mathrm{~h}$ significantly increased the percentage of MB49 cells in the G1 phase $(p<0.01$ and $p<0.001$, respectively; Figure 2A), while decreasing the percentage in the $S$ phase $(p<0.05)$. These results suggest that GFW treatment leads to cell cycle arrest in the G1 phase. After administration of 1 and $2 \mathrm{mg} / \mathrm{mL}$ GFW, we observed a significant increase in the proportion of MB49 cells in the G1 phase. These values are correlated with the reduction in the proportion in the $S$ phase at these concentrations.

We also observed that treating MB49 cells with $2 \mathrm{mg} / \mathrm{mL}$ for $24 \mathrm{~h}$ induced cellular apoptosis, as evidenced by the sub-G1 population, which was proportionately larger than that of the untreated control (Figure 2A). We subsequently assessed the effects of GFW treatment at concentrations of 0.5 , 1 and $2 \mathrm{mg} / \mathrm{mL}$ for $24 \mathrm{~h}$ on the induction of apoptosis. Our results revealed a significant increase in the instances of apoptosis among cells treated with GFW at concentrations of 1 and $2 \mathrm{mg} / \mathrm{mL}$ (Figure 2B). GFW treatment at 1 and $2 \mathrm{mg} / \mathrm{mL}$ (Figure 2B) led to a proportional increase in the number of necrotic cells; however, we observed far fewer necrotic cells than apoptotic cells. We subsequently assessed the effects of GFW treatment at a concentration of $2 \mathrm{mg} / \mathrm{mL}$ over various durations $(0,24,32$ and $48 \mathrm{~h})$ on the induction of caspase- 3 and its cleaved form, which is a hallmark of apoptosis in MB49 cells. The results of Western blot analysis (Figure 2C) revealed a significant increase in the level of cleaved caspase-3 after GFW treatment for $48 \mathrm{~h}$. In the context of the flow cytometry profile, which revealed significant increases in sub-G1 and apoptotic populations, these results demonstrate that GFW induced apoptosis in MB49 cells.

\section{GFW Increases Intracellular ROS Levels and Activates ATM/CHK2 Pathway in the Mediation of Cell Cycle Regulatory Proteins in MB49 Cells}

One promising chemotherapeutic strategy involves dramatically increasing intracellular ROS. This approach has been shown to induce random damage (24-28) and/or apoptosis (29-31) in cancer cells, with little or no effect on normal cells, because of their lower basal ROS levels, which renders them less susceptible to the adverse effects brought about by chemotherapeutic agents. To determine if GFW-mediated cell cycle arrest and apoptosis could be attributed to the generation of ROS, we treated MB49 cells with GFW for $3 \mathrm{~h}$ and then measured the ROS levels. As shown in Figure 3A, the mean percentages of ROS-positive cells (M2) were as follows: untreated control group, $2.1 \pm 0.7 \% ; \mathrm{H}_{2} \mathrm{O}_{2}$ treatment group, $4.2 \pm$ $0.1 \%$; and GFW treatment group, $12.6 \pm$ $0.9 \%$. The GFW-induced accumulation of ROS was three times higher than that induced by $\mathrm{H}_{2} \mathrm{O}_{2}$. These results indicate that GFW enhances the intracellular accumulation of ROS in MB49 cells.

Previous studies have demonstrated that the expression of ATM induced by $\operatorname{ROS}(22,32)$ can mediate cell cycle checkpoints through the activation of CHK2 $(33,34)$. Thus, we examined the induction of ATM, CHK2 and Thr68-phosphorylated CHK2 protein levels in GFW-treated MB49 cells. After treatment with GFW $(2 \mathrm{mg} / \mathrm{mL})$ for various durations, cell lysates were prepared for detection by Western blot analysis by using antibodies specific to ATM, CHK2 and Thr68-phosphorylated CHK2. As shown in Figure 3B, we observed a significant increase in ATM expression in MB49 cells after treatment for $6 \mathrm{~h}$. This step was immediately followed by the activation of CHK2, as indicated by a significant increase in Thr68-phosphorylated CHK2 protein levels. The concurrent use of antibodies specific to P21, P53 and Ser15-phosphorylated P53 prompted a significant increase in the levels of P21 and Ser15-phosphorylated P53 protein in MB49 cells, following GFW treatment for $6 \mathrm{~h}$ (Figure 3C). These results indicate that GFW induces the generation of ROS and the subsequent activation of ATM/CHK2 signaling leading to the induction of Ser15-phosphorylated P53 and P21.

\section{GFW Inhibits Tumor Growth in a Murine Orthotopic Bladder Tumor Model}

Our results in a previous study suggested that high concentrations of GFW in vitro could have cytotoxic effects on normal urothelial cells (15). In view of the fact that high concentrations of GFW were used in this in vivo study, we sought to determine whether GFW has cytotoxic effects on the urothelium of normal mouse bladders. Hematoxylin and eosin (H\&E) staining was used to observe mouse bladder tissue after 10 consecutive GFW treatments with or without $\mathrm{AgNO}_{3}$ chemical lesions. All cases revealed bladders with a normal appearance and regular urothelium. Even 10 consecutive treatments with $180 \mathrm{mg} / \mathrm{mL}$ GFW was insufficient to harm a normal mouse bladder urothelium (Figure 4A). Consequently, GFW was applied at a concentration of $180 \mathrm{mg} / \mathrm{mL}$ in all subsequent in vivo experiments.

Orthotopic bladder tumors were then generated in 35 mice that were randomly assigned to five groups (seven mice per group). Except for the control group (positive, Supplementary Figure S3), which showed a slight decrease in body weight from $10 \mathrm{~d}$ after tumor implantation, all other groups presented a steady body weight throughout the experiment (Supplementary Figure S2). After the implantation of MB49 murine bladder cancer cells for 9-11 d, hematuria was observed in all mice, regardless of whether they underwent treatment (Table 1). This result demonstrates the successful implantation of MB49 cells and subsequent growth of tumors. It is interesting to note that serious hematuria was observed 


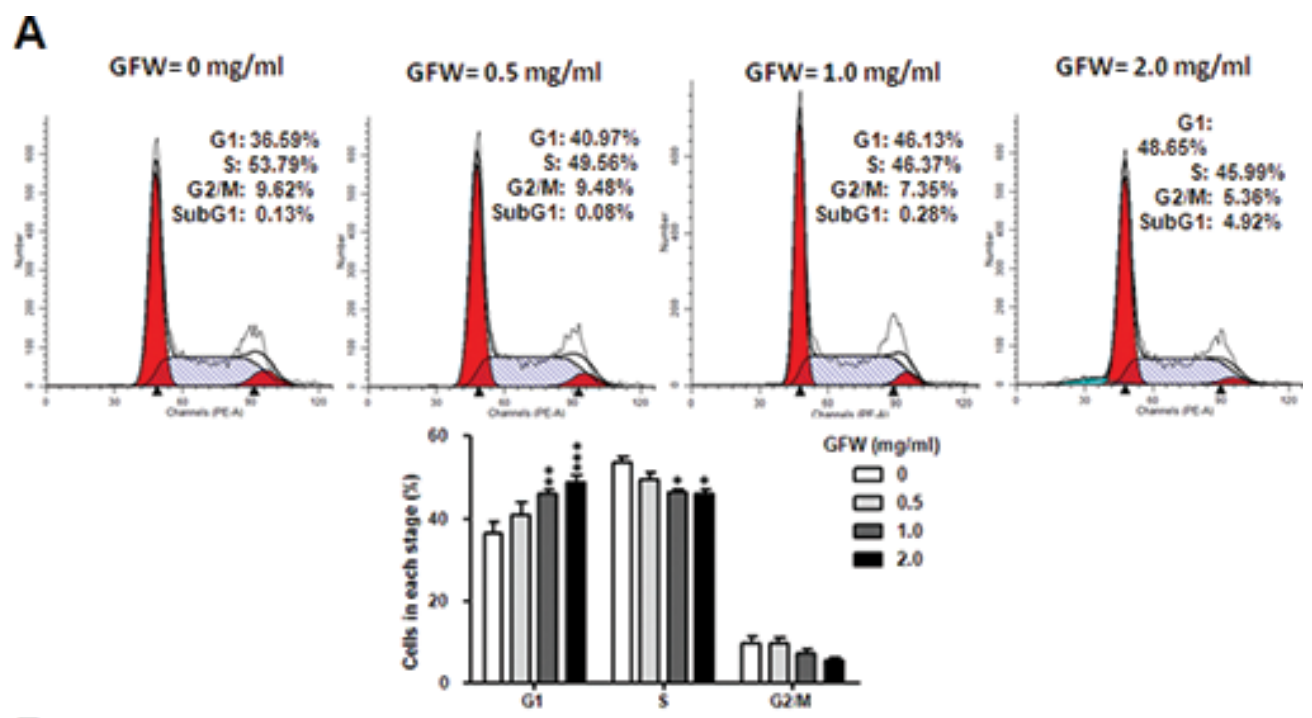

B
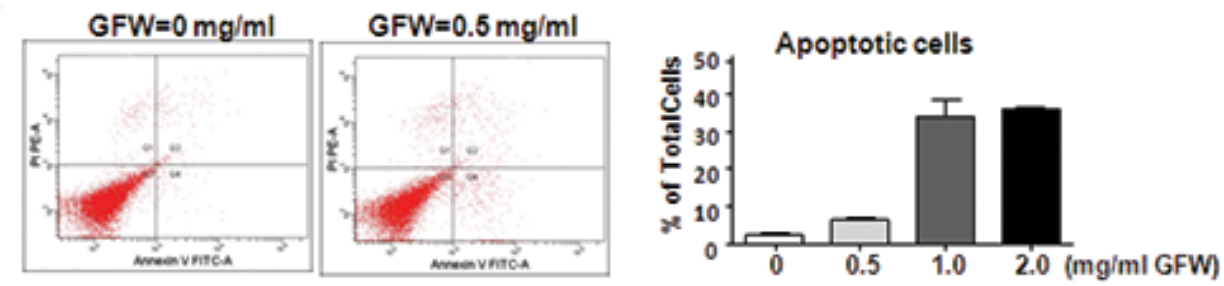

$\mathrm{GFW}=1 \mathrm{mg} / \mathrm{ml}$

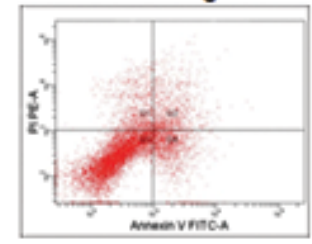

$\mathrm{GFW}=2 \mathrm{mg} / \mathrm{ml}$
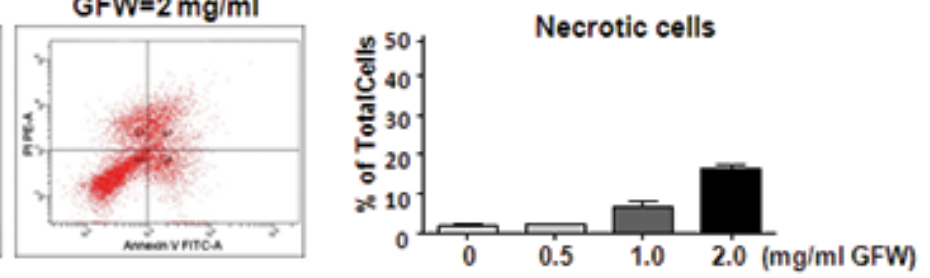

C

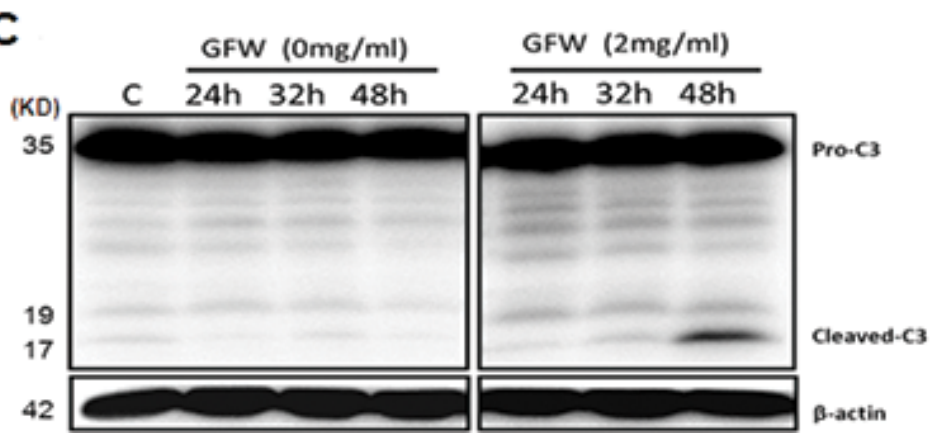

Figure 2. Effects of GFW on cell cycle and induction of apoptosis in mouse urothelial cell line, MB49. (A) MB49 cells were treated with GFW at concentrations of $0.5,1$ and $2 \mathrm{mg} / \mathrm{mL}$ for $24 \mathrm{~h}$ and then stained with propidium iodide and analyzed using flow cytometry. Data are represented as mean $\pm \operatorname{SEM}(n=4)$. Significant differences from the control are indicated by ${ }^{*} p<0.05,{ }^{* *} p<0.01$ or ${ }^{* * *} p<0.001$, as determined by one-way ANOVA and Dunnett comparison test. (B) Analysis of apoptosis using flow cytometry. MB49 cells were treated with GFW at concentrations of $0.5,1$ and $2 \mathrm{mg} / \mathrm{mL}$ for $24 \mathrm{~h}$, stained with propidium iodide and Annexin $V$ and then analyzed using flow cytometry. The lower left quadrant (Q3) represents viable cells, the upper left quadrant (Q1) represents necrotic cells, the lower right quadrant (Q4) represents early apoptotic cells and the upper right quadrant (Q2) represents nonviable late apoptotic cells. The relative percentages include early and late apoptotic cells. Data are represented as mean \pm SEM of three independent experiments. Significant differences from the untreated control are indicated by ${ }^{*} p<0.05,{ }^{* *} p<0.01$ or ${ }^{* * *} p<0.001$, as determined by one-way ANOVA and Dunnett comparison test. (C) Analysis of caspase-3 activation in GFW-treated cells. Western blot analysis was performed to detect caspase-3 and its cleaved form in MB49 cells after treatment with $2 \mathrm{mg} / \mathrm{mL}$ GFW for various durations. $\beta$-Actin protein was used as the loading control. 
A
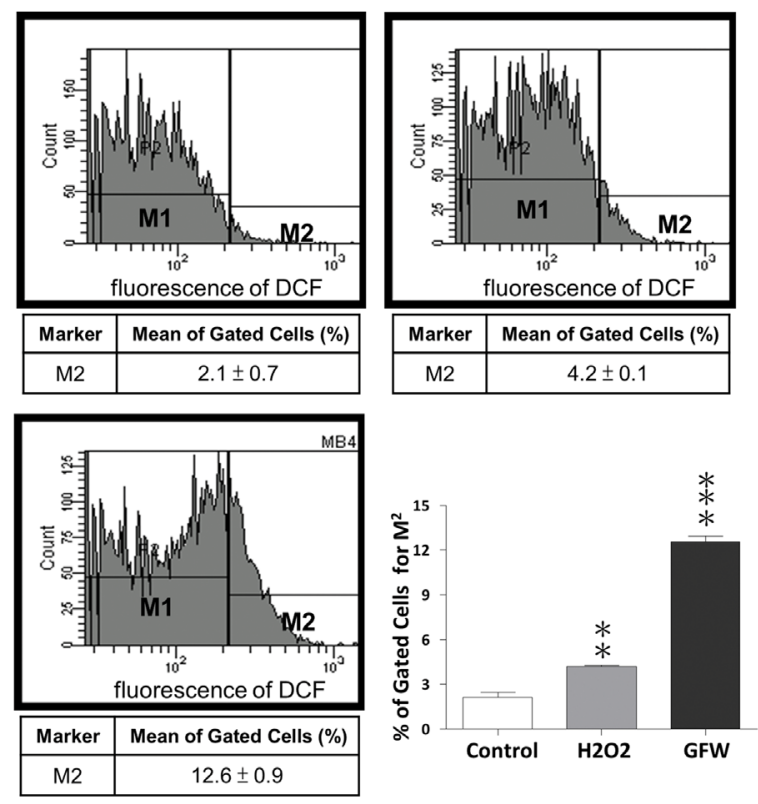

B

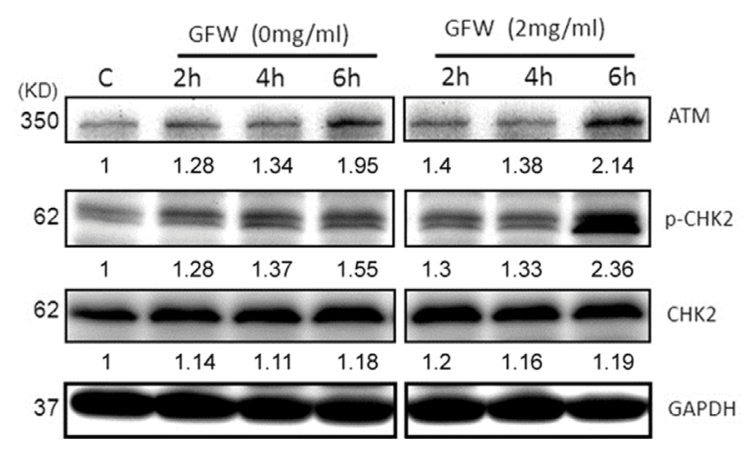

C

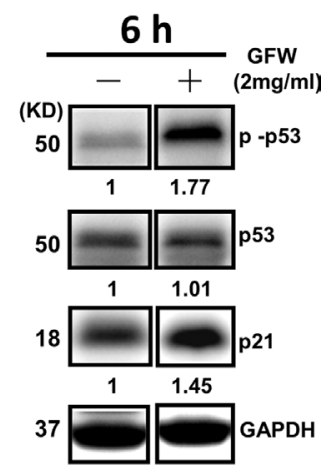

Figure 3. GFW enhanced ROS accumulation and activation of ATM/CHK2 and ATM/P53 signaling in MB49 cells. (A) MB49 cells were pre-incubated with DCFH-DA for $1 \mathrm{~h}$, followed by treatment with $2 \mathrm{mg} / \mathrm{mL} \mathrm{GFW}$ or $50 \mu \mathrm{mol} / \mathrm{L} \mathrm{H}_{2} \mathrm{O}_{2}$ for $3 \mathrm{~h}$. Cells were then examined using flow cytometry to identify DCF fluorescent cells. Data are represented by M1 and M2 percentage of fluorescent cells. M1 is placed around the untreated cells. M2 is placed to the right of $\mathrm{Ml}$ as a designation of positive events. The mean $\pm \operatorname{SEM}(n=3)$ of percentage gated for M2 is shown. Significant differences from the untreated controls are indicated by ${ }^{* *} p \leq 0.01$ or ${ }^{* * *} p \leq 0.001$, as determined by one-way ANOVA and Dunnett comparison test. (B) MB49 cells untreated versus cells treated with GFW for 2, 4 and $6 \mathrm{~h}$ were examined for levels of ATM, CHK2 and Thr68-phosphorylated CHK2 by using Western blot analysis. (C) The expression levels of P21, P53 and Ser15-phosphorylated P53 were subsequently quantified in MB49 cells that did or did not undergo GFW treatment for $6 \mathrm{~h}$. GAPDH protein was used as a loading control. Numbers indicate band intensities as determined by ImageJ software (National Institutes of Health; http://rsb.info.nih.gov/ij/).

in all mice treated with Mito-C or BCG throughout the study period, whereas only mild hematuria was observed in GFW-treated mice, which disappeared by d 17 (Table 1, Figure 4B). These results indicate that GFW significantly reduced the gross/macroscopic hematuria caused by tumor growth. The monitoring of tumor growth was performed by using MRI on d 0, 4, 7 and 11 after GFW treatment. On d 7, a number of tumors were observed in the animals that had not undergone any treatment (Figure 5, upper panel, $\mathrm{C}$ group), whereas fewer tumors were observed in the animal that did undergo treatment, even on the 11th day (Figure 5, lower panel, GFW group). Importantly, tumors treated with GFW presented ATM (Supplementary Figure S3) and p53 (Supplementary Figure S4) activation similar to that observed in cell line experiments. These findings demonstrate that GFW inhibited tumor growth in vivo.

On the final day of treatment, bladder volume was measured to compare the antitumor effects of intravesical treatment with GFW, BCG and mitomycin C. The mean bladder volumes in mice with or without intravesical PBS wash were $371.7 \pm 335.1$ and $445.1 \pm 208 \mathrm{~mm}^{3}$, respectively. The mice treated with GFW, BCG and Mito-C presented mean bladder volumes of $95.1 \pm 66,168.7 \pm 123.3$ and $51.9 \pm 38.6 \mathrm{~mm}^{3}$, respectively (Figure 6A, Table 1). Note that the GFW, BCG and Mito-C treatment groups did not differ significantly with regard to mean bladder volume. All three groups presented volumes far lower than those in mice that did not undergo treatment, which is a clear indication that GFW is at least as effective as BCG and Mito-C in the inhibition of bladder tumor growth.

We subsequently used H\&E staining for pathologic analysis of the urothelial wall of mouse bladders. As shown in Figure 6B, the urothelial wall of bladders in the GFW treatment group appear normal, whereas tumor cells can be observed in the control, BCG treatment, Mito-C treatment and PBS groups. Tumor cells were identified in approximately one in seven GFW-treated mice (14.3\%), seven out of seven BCG-treated mice $(100 \%)$ and two out of seven Mito-C-treated mice $(28.6 \%)$ (Table 1$)$, thereby demonstrating that intravesical treatment with GFW was the most effective treatment with regard to tumor suppression. Mouse kidney tissue was also subjected to pathologic analysis to determine whether metastasis or injury had occurred in the kidney after 
A

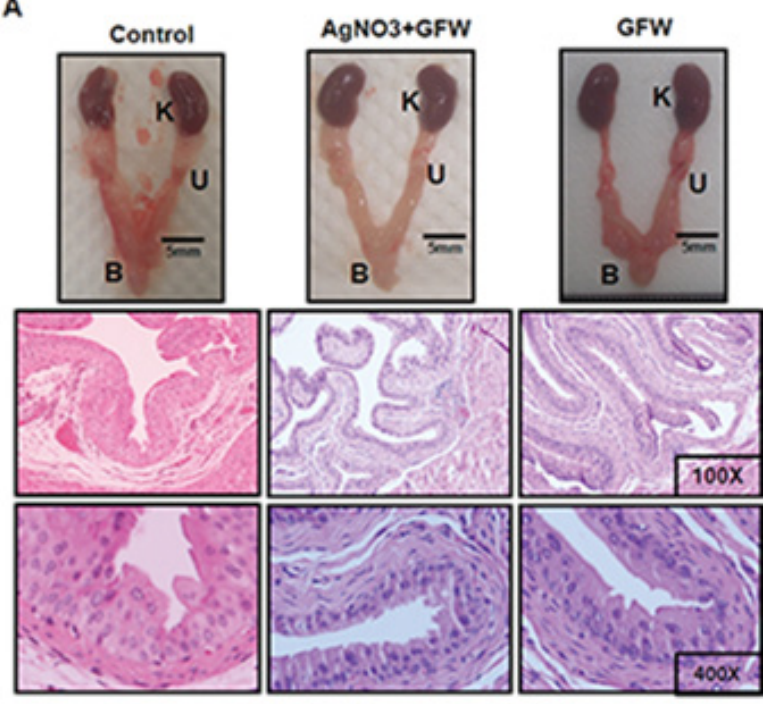

B
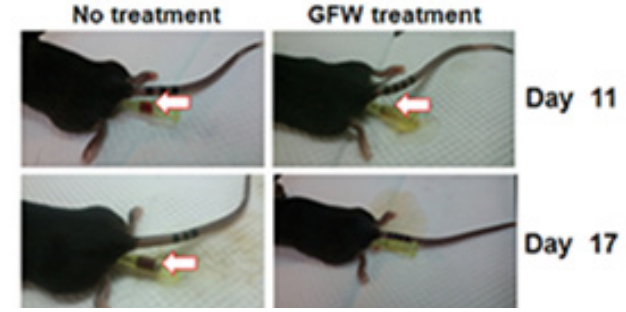

Figure 4. GFW is harmless to normal bladder urothelium of C57BL/6 mice and reduces the hematuria caused by tumor growth in mouse bladders. (A) Female C57BL/6 mice aged 6 wks with no tumor implantation underwent treatment with PBS or GFW $(180 \mathrm{mg} / \mathrm{mL})$ 10 times, administered every other day. "AgNO3+GWF" indicates GFW-treated mice with initial AgNO3-induced chemical lesions on bladder urothelium. After the mice were sacrificed, the urinary system was isolated and photographed (upper panel). K, U and B indicate the kidney, ureter and bladder, respectively. Bladder sections were stained with H\&E for histopathology. Magnification: 100x (middle panel) and 400x (bottom panel). In all cases, the bladders presented a normal appearance and uniform urothelium, indicating that even 10 consecutive treatments with GFW was insufficient to harm the urothelium of normal mouse bladders. (B) After implantation of MB49 murine bladder cancer cells, urine was inspected and photographed. Serious events of hematuria were observed in all of the untreated control mice on $\mathrm{d} 11$ and 19, while only mild hematuria was observed in GFW-treated mice on d 11. This result indicates that GFW reduced the gross hematuria caused by tumor growth in mouse bladder. The arrow indicates hematuria.

intravesical application of the drugs. Pathologic data failed to reveal tumor cells or lesions in any of the kidney sections (Supplementary Figure S5). These results indicate that metastasis had not occurred in our murine orthotopic bladder tumor model, indicating that intravesical treatment with these agents is harmless to the kidneys of orthotopic mice.

\section{DISCUSSION}

This study demonstrated the ability of GFW to suppress bladder cancer in vitro as well as in vivo. The anticancer activity of GFW appears to be associated with the inhibition of cancer cell growth via the induction of apoptosis and cell cycle arrest in the G1 phase. To reveal the effects of GFW on cell cycle progression in MB49 cells, we investigated the accumulation of intracellular ROS, the activation status of the ATM/CHK2 pathway and the expression levels of cell cycle regulatory protein P21. Our results indicated a correlation between GFW-induced G1 arrest in MB49 cells and P21 induction, which depends on ROS-induced activation of the ATM/CHK2 pathway (summarized in Figure 6C). This result is supported by the fact that intracellular ROS levels increased significantly in MB49 cells in response to GFW treatment for $3 \mathrm{~h}$ (Figure 3A). The increase in Thr68-phosphorylated CHK2 at $6 \mathrm{~h}$, followed by an immediate increase in p21 expression, is a clear indication of the rapid, transient activation of ATM/CHK2 (Figure 3B). P21 is known to inhibit the activation of cyclin-dependent protein kinase

Table 1. Parameters in orthotopic bladder tumor model with and without intravesical therapy.

\begin{tabular}{|c|c|c|c|c|c|c|}
\hline & Control & GFW & BCG & Mito-C & PBS & $P^{a}$ \\
\hline Hematuria began (days postimplantation) & $9.6 \pm 1$ & $9.3 \pm 0.8$ & $9.9 \pm 0.4$ & $9.0 \pm 0$ & $9.6 \pm 1$ & 0.1854 \\
\hline Hematuria ended (days postimplantation) & $>22$ & 17 & $>25$ & $>22$ & $>22$ & \\
\hline Body weight (g) at the beginning & $21.7 \pm 2.5$ & $21.9 \pm 1.8$ & $22.8 \pm 3.4$ & $20.7 \pm 1.3$ & $20.2 \pm 1.6$ & 0.2515 \\
\hline Body weight $(g)$ at the end & $19.0 \pm 3.9$ & $22.7 \pm 1.2$ & $20.5 \pm 2.9$ & $20.3 \pm 1.6$ & $19.7 \pm 1.3$ & 0.0774 \\
\hline Bladder volume $\left(\mathrm{mm}^{3}\right)$ at the end & $445.1 \pm 208$ & $95.1 \pm 66$ & $168.7 \pm 123.3$ & $51.9 \pm 38.6$ & $371.7 \pm 335.1$ & 0.0012 \\
\hline Animals with tumor cells identified in bladder ${ }^{b}$ & $7(7)$ & $1(7)$ & $7(7)$ & $2(7)$ & $7(7)$ & \\
\hline
\end{tabular}

aANOVA.

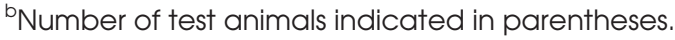




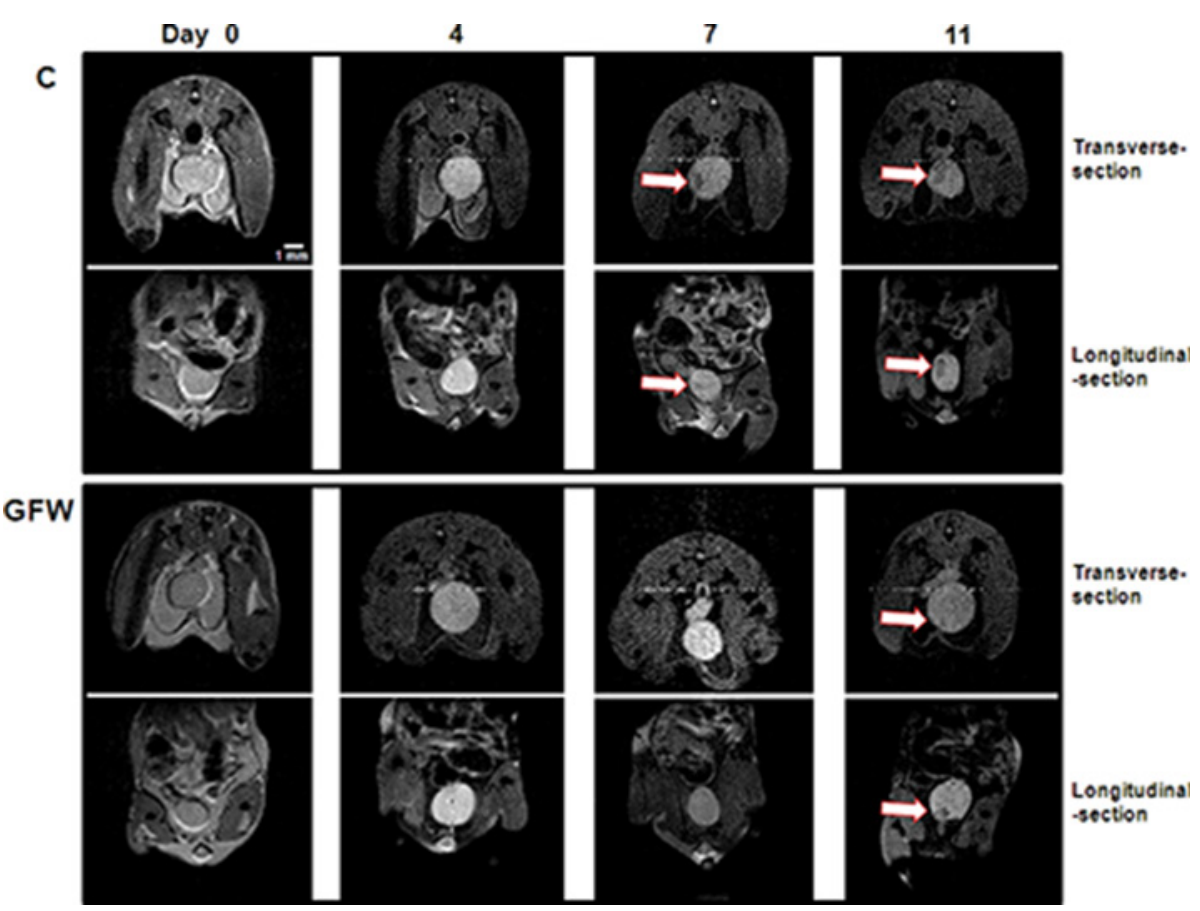

Figure 5. In vivo magnetic resonance detection of bladder tumor growth. Detection of bladder tumors was performed by using MRI on $d 0,4,7$ and 11 after implantation of murine bladder cancer cells. A transverse section and longitudinal section of the MRI results were photographed. These images show tumors in the untreated control mice observed on $\mathrm{d} 7$ and 11 (upper panel, $\mathrm{C}$ group), while a relative small tumor was observed in the GFW-treated mice on d 11 (the lower panel, GFW group). This demonstrates the retardation of tumor growth by GFW. Arrows indicate bladder tumors. Scale bar, $1 \mathrm{~mm}$.

Cdk2, leading to cell cycle arrest at the G1/S transition (35). Previous studies have also revealed the involvement of ATM/CHK2 signaling in the regulation of cell cycle progression in the G1/S phase (36) via the upregulation of P21 gene expression (37).

It has been demonstrated that cell cycle arrest can lead to the induction of apoptosis $(38,39)$. In this study, MB49 cells consistently underwent GFW-induced apoptosis, as evidenced by the presence of a sub-G1 population (Figure 2A), a dose-dependent increase in apoptotic cells (Figure 2B) and an increase in the level of activated caspase-3 after treatment (Figure 2C). These findings are a clear indication that GFW is a potent inducer of apoptosis. ATM activation is responsible for the induction of ser15-phosphorylated P53 (40), leading to caspase-mediated apoptosis (41). One current study has reported that Gypenosides, an active component isolated from the Chinese herb Gynostemma pentaphyllum (Thunb.) Makino, can induce apoptosis in human oral cancer SAS cells via ATM/ATR pathway activation, p53 phosphorylation and caspase- 3 activation (42). Similarly, we detected an increase in the level of ATM, Ser15-phosphorylated p53 in MB49 cells after GFW treatment for $6 \mathrm{~h}$ (Figures 3B, C) and subsequent caspase-3 cleavage after GFW treatment for 32-48 h (Figure 2C). These results suggest that GFW induces the ATM/P53/caspase-3 signal transduction pathway, resulting in MB49 cell apoptosis (summarized in Figure 6C). Annexin V/propidium iodide flow cytometry analysis also revealed a significant increase in necrosis in MB49 cells after GFW treatment (Figure 2B). This result may be due to GFW-induced activation of ATM/CHK2 signaling with the subsequent induction of P21 expression resulting in premature senescence. This scenario has previously been suggested to play a role in tumor suppression by reducing the replicative potential of cells (43). These effects are frequently detected in tumors obtained from patients who underwent genotoxic chemotherapy (44).

This study implemented a murine orthotopic bladder tumor model to analyze the in vivo effects of intravesical GFW therapy. In addition to inhibiting tumor growth in the bladder (Figure 5, Figure 6A), GFW also proved to be more effective than Mito-C and BCG as an intravesical agent in reducing hematuria associated with bladder tumor growth (Table 1). Notably, our pathologic data revealed that tumor cells appeared with far greater frequency in the BCG-treated group than in the Mito-C- and GFW-treated groups (100\% versus $28.6 \%$ and $100 \%$ versus $14.3 \%$, respectively; Figure 6B, Table 1), despite the similar effects of these three agents in inhibiting bladder tumor growth (Figure 6A). Interestingly, a formal meta-analysis of studies on comparative toxicity provided solid evidence to support the assertion that cystitis occurs more frequently among patients receiving BCG than among those administered Mito-C (53.8\% versus $39.2 \%$ ) (45). Nonetheless, we were unable to identify a significant number of lesions on mouse bladder tissue after 10 consecutive treatments with a high concentration of GFW (Figure 4A). This result is a demonstration of the low toxicity of GFW as an intravesical agent against bladder cancer. Moreover, intravesical therapy involves the local administration of the drug, thereby allowing direct contact between therapeutic agents and urothelial tumor. In cases where the bladder tumor is superficial, the direct application of GFW may provide more effective anticancer effects than would be possible with the oral administration of GFW. Finally, no cases of kidney 
A

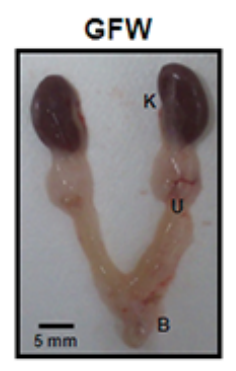

C

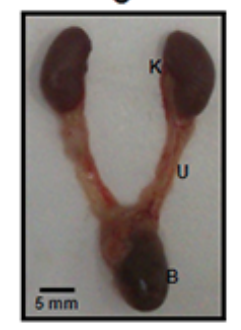

BCG

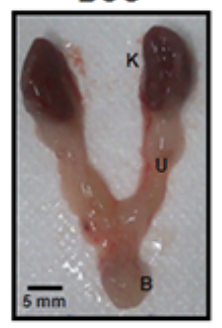

PBS

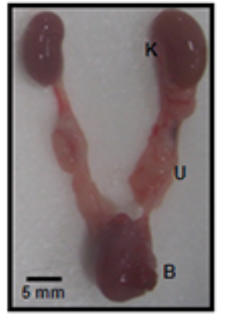

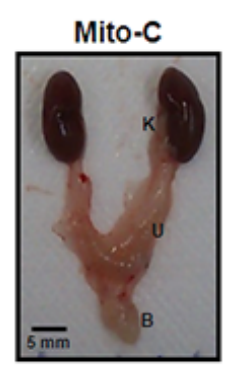

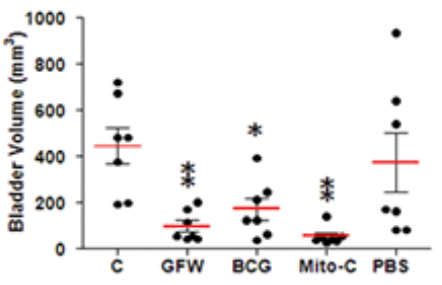

B
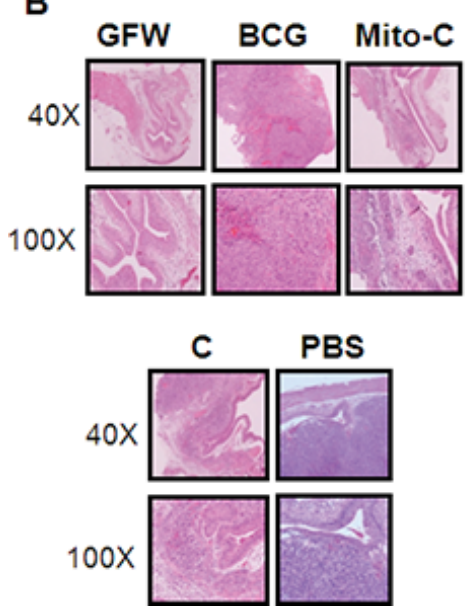

C

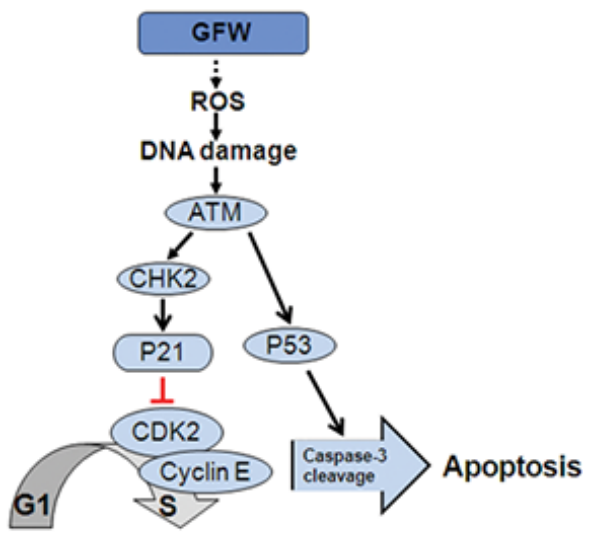

Figure 6. Effect of intravesical therapy in murine orthotopic bladder tumor model. (A) Female C57BL/6 mice aged 6 wks with tumor implantation were untreated (C) or treated with GFW, BCG, Mito-C or PBS according to the time schedule in Figure 1. After the mice were sacrificed, the urinary system was isolated and photographed. K, U and B indicate the kidney, urethra and bladder, respectively. The bladder volume was calculated by (length $\times$ width $\left.^{2}\right) / 2$. Data are presented as mean $\pm \operatorname{SEM}(n=7)$. Significant differences from the control are indicated by ${ }^{*} p<0.05,{ }^{* *} p<0.01$, as determined by one-way ANOVA and Dunnett comparison test. (B) After the treatment cycle, the mice were sacrificed and bladder sections were stained with H\&E for histopathologic examination. Magnification: 40x (upper panel) and 100x (bottom panel). The urothelium in GFWtreated mice presented a normal uniform appearance. Tumor cells were observed only in the untreated control, BCG-treated, Mito-C-treated and PBS-treated mice. (C) Schematic diagram showing the effects of GFW on cell cycle arrest and apoptosis in MB49 murine bladder cancer cells. Intracellular ROS levels in MB49 cells presented a significant increase in response to GFW treatment with a subsequent rapid, transient activation of ATM/CHK2, followed by an immediate increase in P21 expression. P21 suppressed the activation of cyclin-dependent protein kinase Colk2, leading to cell cycle arrest at the G1/S transition. In contrast, GFW induced apoptosis in murine bladder cancer MB49 cells via ATM activation, p53 phosphorylation and caspase-3 activation.

metastasis were observed in our murine orthotopic bladder tumor model, indicating that intravesical treatment with GFW, BCG or Mito-C is harmless to the kidneys of orthotopic mice (Supplementary Figure S2). Similar results were observed in the liver, lung and abdominal cavity of these mice.

\section{CONCLUSION}

The results of this study provide insight into the mechanisms underlying the effects of GFW on mouse bladder cancer cell MB49. We also evaluated the intravesicular application of GFW for the treatment of bladder cancer in vivo. Our data support the further investigation of GFW as a potential agent in the intravesical therapy of superficial bladder cancer.

\section{ACKNOWLEDGMENTS}

This study was supported by the research grant of Ditmanson Medical Foundation Chia-Yi Christian Hospital, Chia-Yi, Taiwan, ROC (R102-22), and the Ministry of Science and Technology, Taiwan, ROC (MOST 104-2320-B-194-003, MOST 104-2314-B-194-001-MY3). The authors would like to thank Dr. Yu-Wen Chen at the Instrument Center of the National Chung Cheng University for the support in MRI and Ditmanson Medical Foundation Chia-Yi Christian Hospital Institutional Review Board for their support (IRB no. 099078).

\section{DISCLOSURE}

The authors declare that they have no competing interests as defined by Molecular Medicine, or other interests that might be perceived to influence the results and discussion reported in this paper.

\section{REFERENCES}

1. Roupret M, et al. (2011) European guidelines for the diagnosis and management of upper urinary tract urothelial cell carcinomas: 2011 update. Eur. Urol. 59:584-94.

2. Shen CH, et al. (2010) Combretastatin A-4 inhibits cell growth and metastasis in bladder cancer cells and retards tumour growth in a murine orthotopic bladder tumour model. Br. J. Pharmacol. 160:2008-27. 
3. Jacobs BL, Lee CT, Montie JE. (2010) Bladder cancer in 2010: how far have we come? CA Cancer J. Clin. 60:244-72.

4. Damrauer JS, et al. (2014) Intrinsic subtypes of high-grade bladder cancer reflect the hallmarks of breast cancer biology. Proc. Natl. Acad. Sci. U. S. A. 111:3110-5.

5. Herr HW. (1997) Natural history of superficial bladder tumors: 10- to 20-year follow-up of treated patients. World J. Urol. 15:84-8.

6. Hall MC, et al. (2007) Guideline for the management of nonmuscle invasive bladder cancer (stages Ta, T1, and Tis): 2007 update. J. Urol. 178:2314-30.

7. Barocas DA, Clark PE. (2008) Bladder cancer. Cur. Opin. Oncol. 20:307-14.

8. Ulker V, Apaydin E, Gursan A, Ozyurt C, Kandiloglu G. (1996) Eosinophilic cystitis induced by mitomycin-C. Int. Urol. Nephrol. 28:755-9.

9. Lopez-Beltran A, Luque RJ, Mazzucchelli R, Scarpelli M, Montironi R. (2002) Changes produced in the urothelium by traditional and newer therapeutic procedures for bladder cancer. J. Clin. Pathol. 55:641-7.

10. Hidoussi A, et al. (2007) Eosinophilic cystitis induced by bacillus Calmette-Guerin (BCG) intravesical instillation. Urology. 70:591. e599-10.

11. Betz SA, See WA, Cohen MB. (1993) Granulomatous inflammation in bladder wash specimens after intravesical bacillus Calmette-Guerin therapy for transitional cell carcinoma of the bladder. Am. J. Clin. Pathol. 99:244-8.

12. Park WH, Joo ST, Park KK, Chang YC, Kim CH. (2004) Effects of the Geiji-Bokryung-Hwan on carrageenan-induced inflammation in mice and cyclooxygenase-2 in hepatoma cells of HepG2 and Hep3B. Immunopharmacol. Immunotoxicol. 26:103-12.

13. Park WH, Lee SK, Oh HK, Bae JY, Kim CH. (2005) Tumor initiation inhibition through inhibition COX-1 activity of a traditional Korean herbal prescription, Geiji-Bokryung-Hwan, in human hepatocarcinoma cells. Immunopharmacol. Immunotoxicol. 27:473-83.

14. Yao Z, Shulan Z. (2008) Inhibition effect of Guizhi-Fuling-decoction on the invasion of human cervical cancer. J. Ethnopharmacol. 120:25-35.

15. Lu CC, et al. (2013) The investigation of a traditional Chinese medicine, Guizhi Fuling Wan (GFW) as an intravesical therapeutic agent for urothelial carcinoma of the bladder. BMC Complement. Altern. Med. 13:44.

16. Li Q, et al. (2011) SFE-CO2 extract from Typhonium giganteum Engl. tubers, induces apoptosis in human hepatoma SMMC-7721 cells involvement of a ROS-mediated mitochondrial pathway. Molecules. 16:8228-42.

17. Chen J, Wong HS, Ko KM. (2014) Ursolic acid-enriched herba cynomorii extract induces mitochondrial uncoupling and glutathione redox cycling through mitochondrial reactive oxygen species generation: protection against menadione cytotoxicity in h9c2 cells. Molecules. 19:1576-91.

18. Duan D, Zhang B, Yao J, Liu Y, Fang J. (2014) Shikonin targets cytosolic thioredoxin reductase to induce ROS-mediated apoptosis in human promyelocytic leukemia HL-60 cells. Free Radic. Biol. Med. 70:182-93.

19. Fridovich I. (1999) Fundamental aspects of reactive oxygen species, or what's the matter with oxygen? Ann. N. Y. Acad. Sci. 893:13-8.

20. Fang YZ, Yang S, Wu G. (2002) Free radicals, antioxidants, and nutrition. Nutrition. 18:872-9.

21. Macip S, et al. (2003) Influence of induced reactive oxygen species in p53-mediated cell fate decisions. Mol. Cell. Biol. 23:8576-85.

22. Guo Z, Kozlov S, Lavin MF, Person MD, Paull TT. (2010) ATM activation by oxidative stress. Science. 330:517-21.

23. Chatterjee S, Kundu S, Sengupta S, Bhattacharyya A (2009) Divergence to apoptosis from ROS induced cell cycle arrest: effect of cadmium. Mutat. Res. 663:22-31.

24. Imlay JA, Chin SM, Linn S. (1988) Toxic DNA damage by hydrogen peroxide through the Fenton reaction in vivo and in vitro. Science. 240:640-2.

25. Maynard S, Schurman SH, Harboe C, de SouzaPinto NC, Bohr VA. (2009) Base excision repair of oxidative DNA damage and association with cancer and aging. Carcinogenesis. 30:2-10.

26. Wiseman H, Halliwell B. (1996) Damage to DNA by reactive oxygen and nitrogen species: role in inflammatory disease and progression to cancer. Biochem. J. 313:17-29.

27. Levine RL. (2002) Carbonyl modified proteins in cellular regulation, aging, and disease. Free Rad. Biol. Med. 32:790-6.

28. Wells-Knecht MC, Lyons TJ, McCance DR, Thorpe SR, Baynes JW. (1997) Age-dependent increase in ortho-tyrosine and methionine sulfoxide in human skin collagen is not accelerated in diabetes: evidence against a generalized increase in oxidative stress in diabetes. J. Clin. Invest. 100:839-46.

29. Qanungo S, Das M, Haldar S, Basu A. (2005) Epigallocatechin-3-gallate induces mitochondrial membrane depolarization and caspasedependent apoptosis in pancreatic cancer cells. Carcinogenesis. 26:958-67.

30. Zhang R, Humphreys I, Sahu RP, Shi Y, Srivastava SK. (2008) In vitro and in vivo induction of apoptosis by capsaicin in pancreatic cancer cells is mediated through ROS generation and mitochondrial death pathway. Apoptosis. 13:1465-78.

31. Sahu RP, Zhang R, Batra S, Shi Y, Srivastava SK. (2009) Benzyl isothiocyanate-mediated generation of reactive oxygen species causes cell cycle arrest and induces apoptosis via activation of MAPK in human pancreatic cancer cells. Carcinogenesis. 30:1744-53.

32. Kubota S, et al. (2014) Activation of the prereplication complex is blocked by mimosine through reactive oxygen species-activated ataxia telangiectasia mutated (ATM) protein without DNA damage. J. Biol. Chem. 289:5730-46.

33. Chen Y, Poon RY. (2008) The multiple checkpoint functions of $\mathrm{CHK} 1$ and CHK2 in maintenance of genome stability. Front. Biosci. 13:5016-29.

34. Smith J, Tho LM, Xu N, Gillespie DA. (2010) The ATM-Chk2 and ATR-Chk1 pathways in DNA damage signaling and cancer. Adv. Cancer Res. 108:73-112.

35. Bisteau X, et al. (2013) CDK4 T172 phosphorylation is central in a CDK7-dependent bidirectional CDK4/CDK2 interplay mediated by p21 phosphorylation at the restriction point. PLoS Genet. 9:e1003546.

36. Nikitin PA, Price AM, McFadden K, Yan CM, Luftig MA. (2014) Mitogen-induced B-cell proliferation activates Chk2-dependent G1/S cell cycle arrest. PLoS One. 9:e87299.

37. Xie G, et al. (1998) Requirements for p53 and the ATM gene product in the regulation of $\mathrm{G} 1 / \mathrm{S}$ and S phase checkpoints. Oncogene. 16:721-36.

38. Hartwell LH, Kastan MB. (1994) Cell cycle control and cancer. Science. 266:1821-8.

39. Vermeulen K, Berneman ZN, Van Bockstaele DR. (2003) Cell cycle and apoptosis. Cell Prolif. 36:165-75.

40. Canman CE, et al. (1998) Activation of the ATM kinase by ionizing radiation and phosphorylation of p53. Science. 281:1677-9.

41. Jiang C, Hu H, Malewicz B, Wang Z, Lu J. (2004) Selenite-induced p53 Ser-15 phosphorylation and caspase-mediated apoptosis in LNCaP human prostate cancer cells. Mol. Cancer Ther. 3:877-84.

42. Lu KW, et al. (2010) Gypenosides causes DNA damage and inhibits expression of DNA repair genes of human oral cancer SAS cells. In vivo. 24:287-91.

43. Kahlem P, Dorken B, Schmitt CA. (2004) Cellular senescence in cancer treatment: friend or foe? J. Clin. Invest. 113:169-74.

44. te Poele RH, Okorokov AL, Jardine L, Cummings J, Joel SP. (2002) DNA damage is able to induce senescence in tumor cells in vitro and in vivo. Cancer Res. 62:1876-83

45. Bohle A, Jocham D, Bock PR. (2003) Intravesical bacillus Calmette-Guerin versus mitomycin $C$ for superficial bladder cancer: a formal meta-analysis of comparative studies on recurrence and toxicity. J. Urol. 169:90-5.

Cite this article as: Lu C-C, et al. (2016) Guizhi Fuling Wan as a novel agent for intravesical treatment for bladder cancer in a mouse model. Mol. Med. 22:64-73. 